\title{
ON CIRCLE MAP COUPLED MAP LATTICE
}

\author{
E. AHMED and A. S. HEGAZI
}

Received 27 November 2001

\begin{abstract}
The circle map in one and two dimensions is studied. Both its stability and synchronization, using a bounded control and persistence, are discussed. This work is expected to be applicable in ecology where spatial effects are known to be important. Also, it will be relevant to systems where delay effects are not negligible.
\end{abstract}

2000 Mathematics Subject Classification: 37N25.

1. Introduction. The circle map is one of the subjects which is both mathematically interesting and useful in applications (see [7, 10, 11, 12]). It relates, in a beautiful way, some pure mathematical problems and applications.

As an example, in [7], it is shown that the simplest circle map $f(\theta)=\theta+$ $\tau_{0} \bmod 1$, where $\tau_{0}$ is a constant, is related to a human heart disease (parasystole) where there are two types of heart pulses. This is represented by a symbolic sequence of two symbols (say 0,1 ). Such a sequence defines another one, called the reduced sequence where one starts with 1 and counts the number of iterates till the next 1 appears and so forth. This reduced sequence has remarkable properties with some relevance to number theory: (i) the reduced sequence contains, at most, three integers, say $p, m$, and $n$; (ii) $p=m+n$; (iii) $m$ or $n$ is odd; and (iv) only one of $p, m$, and $n$ can succeed in the reduced sequence. These results agree with clinical observations.

A relation with the number theory can be obtained [11] by counting the number of periodic points for the simple circle map $f(\theta)=a \theta \bmod 2 \pi$ with $a>1$. It is direct to see that the periodic points with a period $n$ are $2 j \pi /\left(a^{n}-\right.$ $1), j=0,1, \ldots, a^{n}-2$. If $a$ is an integer and $n$ is a prime number, and subtracting the number of fixed points, then the number of genuine periodic points with period $n$ is $a^{n}-a$. Thus, $\left(a^{n}-a\right) / n \in \mathbb{N}$. This proves the following theorem of Fermat.

THEOREM 1.1. (i) If $n$ is a prime number for all integer $a$, then $\left(a^{n}-a\right) / n \in \mathbb{N}$.

(ii) If $n$ and a are relatively prime, then $\left(a^{n-1}-1\right) / n \in \mathbb{N}$.

Following the same line of reasoning, let $n=n_{1} n_{2}$, where both $n_{1}$ and $n_{2}$ are prime, then the number of genuine periodic points of the previous circle map with period $n$ is equal to $a^{n}-1-($ the number of fixed points) - (the number of genuine periodic points with period $\left.n_{1}\right)$ - (the number of genuine periodic 
points with period $n_{2}$ ). This number is divisible by $n$, hence

$$
\begin{gathered}
\frac{\left[\left(a^{n}-1\right)-(a-1)-\left(a^{n_{1}}-a\right)-\left(a^{n_{2}}-a\right)\right]}{n} \in \mathbb{N} \\
\quad \Longrightarrow \frac{\left[\left(a^{n_{1}}-a\right)-\left(a^{n_{2}}-a\right)\right]}{n} \in \mathbb{N} \\
\quad \Longrightarrow\left(a^{n_{1}}-a\right)-\left(a^{n_{2}}-a\right)
\end{gathered}
$$

is divisible by both $n_{1}$ and $n_{2}$, thus

$$
\frac{a^{n_{1}}-a}{n_{1}} \in \mathbb{N}, \quad \frac{a^{n_{2}}-a}{n_{2}} \in \mathbb{N} .
$$

The more familiar circle map (called sine circle map) has the form

$$
\theta_{t+1}=a \theta_{t}+\Omega-\left(\frac{k}{2 \pi}\right) \sin \left(2 \pi \theta_{t}\right)
$$

Without loss of generality, set $\Omega \in(0,1)$ and, for most of this note, $a=1$. The equilibrium point equation is

$$
\sin (2 \pi \theta)=\frac{(\Omega+n) 2 \pi}{k},
$$

where $n$ is a chosen integer such that $|2 \pi(\Omega+n) / k|<1$.

The stability analysis implies that the equilibrium point (1.4) is stable if

$$
2 \pi(1-\Omega)<k<2 \sqrt{1+\pi^{2}(1-\Omega)^{2}} .
$$

This agrees with the numerical results (Vandermeer et al. [12]).

Here, different versions of coupled map lattices (CMLs) (see [9]) corresponding to the circle map will be discussed. This is expected to have some applications, for example, in turbulence [4]. The linear stability analysis in [5] will be reviewed and then extended to two dimensions. The synchronization of a circle map using a bounded control is studied. The persistence of CML will be studied and applied to the circle map case. It is an important concept in biology since it determines whether the population will survive or will extinct.

2. Linear stability analysis and synchronization. We consider the CML

$$
\begin{aligned}
& \theta_{j}^{t+1}=(1-D)\left[\theta_{j}^{t}+\Omega-\left(\frac{k}{2 \pi}\right) \sin \left(2 \pi \theta_{j}^{t}\right)\right] \\
&+\left(\frac{D}{2}\right)\left[\left[\theta_{j+1}^{t}+\Omega-\left(\frac{k}{2 \pi}\right) \sin \left(2 \pi \theta_{j+1}^{t}\right)\right]\right. \\
&\left.+\left(\frac{D}{2}\right)\left[\theta_{j-1}^{t}+\Omega-\left(\frac{k}{2 \pi}\right) \sin \left(2 \pi \theta_{j-1}^{t}\right)\right]\right] \bmod 1,
\end{aligned}
$$

where $j=1,2,3, \ldots, n$ and $t=1,2,3, \ldots$. 
Another CML motivated by the work of Zanette [13] is

$$
\theta_{j}^{t+1}=\theta_{j}^{t}+\Omega+D\left\{\sin \left[2 \pi\left(\theta_{j+1}^{t}-\theta_{j}^{t}\right)\right]+\sin \left[2 \pi\left(\theta_{j-1}^{t}-\theta_{j}^{t}\right)\right]\right\} \bmod 1 .
$$

If we include the fact that the interactions have finite velocities, then the CML should be delayed (cf. [13]). In this case, (2.1) is replaced by

$$
\begin{aligned}
& \theta_{j}^{t+1}=(1-D)\left[\theta_{j}^{t}+\Omega-\left(\frac{k}{2 \pi}\right) \sin \left(2 \pi \theta_{j}^{t}\right)\right] \\
&+\left(\frac{D}{2}\right)\left[\left[\theta_{j+1}^{t-1}+\Omega-\left(\frac{k}{2 \pi}\right) \sin \left(2 \pi \theta_{j+1}^{t-1}\right)\right]\right. \\
&\left.+\left(\frac{D}{2}\right)\left[\theta_{j-1}^{t-1}+\Omega-\left(\frac{k}{2 \pi}\right) \sin \left(2 \pi \theta_{j-1}^{t-1}\right)\right]\right] \bmod 1 .
\end{aligned}
$$

In the weak coupling regime ( $D$ is much smaller than 1 ), the relation between the CML dynamics and that of the local systems is given by the following proposition.

Proposition 2.1 (Afraimovich and Fernandez [1]). In the weak coupling limit, the symbolic dynamics of the CML is equivalent to the direct product of the symbolic dynamics of the local subsystems.

Therefore, the stability analysis given in Section 1 will be relevant to the CML case provided that the diffusion constant is small.

Now, an exactly solvable CML model (see [8]) will be given and generalized to the case of delay. The CML

$$
\theta_{j}^{t+1}=a(1-D) \theta_{j}^{t}+\left(\frac{D}{2}\right)\left[\theta_{j+1}^{t}+\theta_{j-1}^{t}\right] \bmod 1
$$

is exactly solvable provided that both $a(1-D)$ and $D / 2$ are integers since, in this case, (2.4) can be written in the form $\theta_{j}^{t+1}=\sum_{k} a_{j k} \theta_{k}^{t} \bmod 1$, where $a_{j k}$ are integers. Hence,

$$
\theta_{j}^{t}=\sum_{k}\left(A^{t}\right)_{j k} \theta_{k}^{0} \bmod 1, \quad A=\left(a_{j k}\right)
$$

The special case $a=1$ in (2.4) corresponds to the case $\Omega=k=0$ in (2.1). Generalizing this result to the case of delayed CML, (2.4) becomes $(a=1)$

$$
\theta_{j}^{t+1}=(1-D) \theta_{j}^{t}+\left(\frac{D}{2}\right)\left[\theta_{j+1}^{t-1}+\theta_{j-1}^{t-1}\right] \bmod 1
$$

Using matrix notation, (2.6) can be written in the form

$$
\underline{\theta}_{t+1}=(I-D) I \underline{\theta}_{t}+B \underline{\theta}_{t-1} \bmod 1,
$$


where $I$ is the $n \times n$ identity matrix, $B=\left(b_{j k}\right)$, and $b_{j k}=(D / 2) \delta_{j, k \pm 1}$. If $D / 2$ is an integer, then

$$
\begin{aligned}
\underline{\theta}_{t}= & {\left[(1-D)^{t-1}+(t-2)(1-D)^{t-3} B+(t-4)(1-D)^{t-5} B^{2}+\cdots\right] \underline{\theta}_{1} } \\
& +\left[(1-D)^{t-2} B+(t-3)(1-D)^{t-4} B^{2}+\cdots\right] \underline{\theta}_{0} \bmod 1,
\end{aligned}
$$

where only positive terms are included in the coefficients of (2.8).

The linear stability analysis of the 1-dimensional circular CML (2.1) has been discussed in [5] around the synchronized solution $\theta_{j}^{t}=$ const for all $j, t$. Here, it is briefly reviewed then generalized. Define $a_{j}^{t}$ and $b_{j}^{t}$ by

$$
a_{j}^{t}=\theta_{j+1}^{t}-\theta_{j}^{t}, \quad b_{j}^{t}=\theta_{j+1}^{t}+\theta_{j}^{t}
$$

then the synchronized solution corresponds to $a_{j}^{t}=0$ and $b_{j}^{t}=s$, where $s$ is given by $(k / \pi) \sin (\pi s)=2 \Omega$. Close to the synchronized solution, the system (2.1) can be approximated by

$$
a_{j}^{t+1}=(1-D)(1-k \cos 2 \pi s) a_{j}^{t}+\left(\frac{D}{2}\right)(1-k \cos 2 \pi s)\left(a_{j+1}^{t}+a_{j-1}^{t}\right) \bmod 1 .
$$

The CML (2.10) or, more generally, any CML of the form

$$
a_{j}^{t+1}=\alpha a_{j}^{t}+\gamma\left(a_{j+1}^{t}+a_{j-1}^{t}\right)
$$

is related to circulant matrices [3]. They have the form

$$
C=\left[\begin{array}{ccccc}
c_{1} & c_{2} & \cdots & \cdots & c_{n} \\
c_{n} & c_{1} & c_{2} & \cdots & c_{n-1} \\
\vdots & \vdots & \vdots & \vdots & \vdots \\
c_{2} & c_{3} & \cdots & \cdots & c_{1}
\end{array}\right]
$$

it can be written in the form $\operatorname{circ}\left(c_{1}, c_{2}, \ldots, c_{n}\right)$. Define the shift matrix $\Sigma=$ $\operatorname{circ}(0,1,0,0, \ldots, 0)$, then it can be shown that $C=\sum_{j=1}^{n} c_{j}(\Sigma)^{j-1}$ and that the eigenvalues of $C$ are given by

$$
\lambda_{r}=\sum_{j=1}^{n} c_{j} \exp \left[\frac{2 \pi i(j-1) r}{n}\right], \quad r=0,1,2, \ldots, n-1
$$


For the CML (2.11), we have $c_{1}=\alpha$ and $c_{2}=c_{n-1}=\gamma$. Thus, the eigenvalues of (2.11) are $\alpha+2 \gamma \cos 2 \pi r / n, r=0,1,2, \ldots, n-1$. Thus, it is clear that the eigenvalues of system (2.10) are

$$
(1-k \cos 2 \pi s)\left[(1-D)+\frac{D \cos 2 \pi r}{n}\right], \quad r=0,1, \ldots, n-1 .
$$

Hence, $\Omega \in(-k /(2 \pi), k /(2 \pi))$ is the stability zone for the synchronized solution of (2.1).

Generalizing to the 2-dimensional case (which is expected to be more relevant to applications), we get

$$
\begin{aligned}
\theta_{i, j}^{t+1}= & (1-D)\left[\theta_{i, j}^{t}+\Omega-\left(\frac{k}{2 \pi}\right) \sin \left(2 \pi \theta_{i, j}^{t}\right)\right] \\
& +\left(\frac{D}{4}\right)\left[\theta_{i, j+1}^{t}+\Omega-\left(\frac{k}{2 \pi}\right) \sin \left(2 \pi \theta_{i, j+1}^{t}\right)\right] \\
& +\left(\frac{D}{4}\right)\left[\theta_{i, j-1}^{t}+\Omega-\left(\frac{k}{2 \pi}\right) \sin \left(2 \pi \theta_{i, j-1}^{t}\right)\right] \\
& +\left(\frac{D}{4}\right)\left[\theta_{i+1, j}^{t}+\Omega-\left(\frac{k}{2 \pi}\right) \sin \left(2 \pi \theta_{i+1, j}^{t}\right)\right] \\
& +\left(\frac{D}{4}\right)\left[\theta_{i-1, j}^{t}+\Omega-\left(\frac{k}{2 \pi}\right) \sin \left(2 \pi \theta_{i-1, j}^{t}\right)\right] \bmod 1 .
\end{aligned}
$$

Generalizing the results of circulant matrices to this case, we get that the eigenvalues are $\alpha \pm 2 \gamma \cos 2 \pi r / n$, where the synchronized solution of (2.15) ( $\theta_{i, j}^{t}=$ const for all $\left.i, j, t\right)$ corresponds to

$$
\begin{gathered}
\alpha=(1-D)(1-k \cos 2 \pi s), \quad \gamma=\left(\frac{D}{4}\right)(1-k \cos 2 \pi s), \\
\left(\frac{k}{\pi}\right) \sin (\pi s)=2 \Omega .
\end{gathered}
$$

Thus, the synchronized solution of (2.15) is stable for all $\Omega \in(-k /(2 \pi)$, $k /(2 \pi))$.

The delayed case is given by the equation

$$
\begin{aligned}
\theta_{i, j}^{t+1}= & (1-D)\left[\theta_{i, j}^{t}+\Omega-\left(\frac{k}{2 \pi}\right) \sin \left(2 \pi \theta_{i, j}^{t}\right)\right] \\
& +\left(\frac{D}{4}\right)\left[\theta_{i, j+1}^{t-1}+\Omega-\left(\frac{k}{2 \pi}\right) \sin \left(2 \pi \theta_{i, j+1}^{t-1}\right)\right] \\
& +\left(\frac{D}{4}\right)\left[\theta_{i, j-1}^{t-1}+\Omega-\left(\frac{k}{2 \pi}\right) \sin \left(2 \pi \theta_{i, j-1}^{t-1}\right)\right] \\
& +\left(\frac{D}{4}\right)\left[\theta_{i+1, j}^{t-1}+\Omega-\left(\frac{k}{2 \pi}\right) \sin \left(2 \pi \theta_{i+1, j}^{t-1}\right)\right] \\
& +\left(\frac{D}{4}\right)\left[\theta_{i-1, j}^{t-1}+\Omega-\left(\frac{k}{2 \pi}\right) \sin \left(2 \pi \theta_{i-1, j}^{t-1}\right)\right] \bmod 1 .
\end{aligned}
$$


Linearizing around the synchronized solution, we get that, in the weak coupling regime, the maximum eigenvalue is $(1-D)(1-k \cos 2 \pi s)$, hence it is stable for all $\Omega \in(-k /(2 \pi), k /(2 \pi))$.

A CML, motivated by the work of Zanette [13], is (2.2). The synchronized solution $\theta_{j}^{t}=$ const independent of $j$ and $t$ requires that $\Omega=0$, and using the previous analysis, we get that the eigenvalues are $1-D+D \cos (2 \pi j / n)$, $j=0,1, \ldots, n-1$.

Now, we discuss Poincaré coupled map lattice (PCML) (see [7]) given by

$$
\theta_{j}^{t+1}=(1-D) g\left(\theta_{j}^{t}\right)+\left(\frac{D}{2}\right)\left[g\left(\theta_{j-1}^{t}\right)+g\left(\theta_{j+1}^{t}\right)\right]
$$

where

$$
g(\theta)=\frac{(a+\cos 2 \pi \theta)}{\sqrt{1+2 a \cos 2 \pi \theta+a^{2}}}, \quad a>0 .
$$

It is known that the function (2.19) has applications in cardiac arrhythmias. Here, the corresponding CML will be studied. The homogeneous equilibrium point is given by

$$
\theta=g(\theta)
$$

and the stability conditions for this equilibrium point are

$$
\left|\left(\frac{1-D-D \cos 2 \pi r}{n}\right) g^{\prime}(\theta)\right|<1, \quad r=0,1, \ldots, n
$$

Now, having found the stability regions, we comment on the synchronization within the chaotic regions (cf. [2]). In the standard approach, two identical systems (master and slave) are given by $x_{t+1}=f\left(x_{t}\right)$ and $y_{t+1}=f\left(y_{t}\right)$, respectively. In general, $x_{t} \neq y_{t}$ due to the sensitive dependence on initial conditions. Then a control $u$ is added to achieve synchronization, hence

$$
x_{t+1}=f\left(x_{t}\right), \quad y_{t+1}=f\left(y_{t}\right)+u_{t}, \quad u_{t}=p\left(f\left(x_{t}\right)-f\left(y_{t}\right)\right), \quad 1 \geq p \geq 0 .
$$

Define $z_{t}=x_{t}-y_{t}$; it is easy to see that if $z_{t}$ is sufficiently small, then the synchronization will occur if

$$
p \geq p_{c}=1-\exp (-\lambda)
$$

where $\lambda$ is the Lyapunov exponent of the map $f$.

This approach has two drawbacks: the first is that (2.23) is valid only if $z_{t}$ is small and the second is that the control $u_{t}$ can be unrealistically large. Therefore, we augment this approach with the condition that only a bounded control is allowed, that is, there exists $u^{*}$ such that if $u_{t}>u^{*}$, then $u_{t}=u^{*}$, if $u_{t}<u^{*}$, then $u_{t}=-u^{*}$. 
We have applied the above procedure to the circle map ( $f$ given by (1.3) with $a=1, k=3.5$, and $\Omega=0.23$ ) and obtained the synchronization for $p \geq 0.54$ and $u^{*}=0.001$. This agrees with the fact that the corresponding Lyapunov exponent is $\lambda=0.539$.

\section{On the persistence of circle map CML}

DEFINITION 3.1. A dynamical system is persistent if, for all $x_{i}(0)>0$,

$$
\liminf _{t \rightarrow \infty} x_{i}(t)>0 \quad \forall i=1,2, \ldots, n
$$

A CML is persistent if all its components are persistent.

An open question is: what is the effect of diffusion on persistence? We consider the following CML:

$$
\theta_{j}^{t+1}=(1-D) \theta_{j}^{t}+\left(\frac{D}{2}\right)\left[\theta_{j+1}^{t}+\theta_{j-1}^{t}\right]+f\left(\theta_{j}^{t}\right)
$$

or

$$
\theta_{j}^{t+1}=(1-D) f\left(\theta_{j}^{t}\right)+\left(\frac{D}{2}\right)\left[f\left(\theta_{j-1}^{t}\right)+f\left(\theta_{j+1}^{t}\right)\right]
$$

Definition 3.2. The set $A$ is an invariant set of the system (3.2) or (3.3) if

$$
\theta_{j}^{0} \in A \forall j=1,2, \ldots, n \Longrightarrow \theta_{j}^{t} \in A \forall j=1,2, \ldots, n, \quad \forall t=1,2,3, \ldots .
$$

The following proposition is a generalization of a result in [6].

Proposition 3.3. (i) If $I=[a, b]$ is in the domain of the function $f$ in (3.2) such that

$$
f(a)=a, \quad f(b)=b, \quad\left(1-D+f^{\prime}(x)\right) \geq 0 \forall x \in I,
$$

then I is an invariant set for system (3.2).

(ii) Similarly for system (3.3) provided that

$$
f^{\prime}(x) \geq 0 \quad \forall x \in I .
$$

Proof. We begin by showing that if

$$
\begin{aligned}
\theta_{j}^{t+1} & \leq(1-D) \theta_{j}^{t}+\left(\frac{D}{2}\right)\left[\theta_{j+1}^{t}+\theta_{j-1}^{t}\right]+f\left(\theta_{j}^{t}\right), \\
\varphi_{j}^{t+1} & \geq(1-D) \varphi_{j}^{t}+\left(\frac{D}{2}\right)\left[\varphi_{j+1}^{t}+\varphi_{j-1}^{t}\right]+f\left(\varphi_{j}^{t}\right), \\
\varphi_{j}^{0} & \geq \theta_{j}^{0}, \quad \forall j=1,2, \ldots, n,
\end{aligned}
$$

then $\varphi_{j}^{t} \geq \theta_{j}^{t}$ for all $j, t$ if $\left(1-D+f^{\prime}(x)\right) \geq 0$ for all $x \in I$. 
For $t=1$, the following is proved:

$$
\begin{aligned}
\varphi_{j}^{1}-\theta_{j}^{1} \geq & \left(\varphi_{j}^{0}-\theta_{j}^{0}\right)+\left(\frac{D}{2}\right)\left[\left(\varphi_{j-1}^{0}-\theta_{j-1}^{0}\right)+\left(\varphi_{j+1}^{0}-\theta_{j+1}^{0}\right)-2\left(\varphi_{j}^{0}-\theta_{j}^{0}\right)\right] \\
& +\left(\varphi_{j}^{0}-\theta_{j}^{0}\right) f^{\prime}\left(\zeta_{j}^{0}\right)
\end{aligned}
$$

where the mean value theorem has been used in the last term. Since $\zeta_{j}^{0} \in$ $\left[\theta_{j}^{0}, \varphi_{j}^{0}\right] \subset I$, then $\varphi_{j}^{1} \geq \theta_{j}^{1}$. The proof for a larger $t$ follows similarly. Now, choose

$$
\theta_{j}^{t}=a, \quad \varphi_{j}^{t}=b, \quad \forall t, j,
$$

then if

$$
\begin{gathered}
\omega_{j}^{t+1}=(1-D) \omega_{j}^{t}+\left(\frac{D}{2}\right)\left[\omega_{j+1}^{t}+\omega_{j-1}^{t}\right]+f\left(\omega_{j}^{t}\right) \Longrightarrow \theta_{j}^{0} \leq \omega_{j}^{0} \leq \varphi_{j}^{0}, \\
\theta_{j}^{t} \leq \omega_{j}^{t} \leq \varphi_{j}^{t},
\end{gathered}
$$

then $I$ is an invariant set for (3.2) provided that

$$
\left(1-D+f^{\prime}(x)\right) \geq 0 \quad \forall x \in I .
$$

The proof of part (ii) is similar.

Proposition 3.4. (i) If there exists $\epsilon>0$ such that, for all $\theta \in[0, \epsilon]$,

$$
f^{\prime}(\theta) \geq 0, \quad\left(1-D+f^{\prime}(\theta)\right) \geq 0,
$$

then the CML (3.2) is persistent.

(ii) If $f(\theta) \geq 0$ for all $\theta \geq 0$ and there exists $\epsilon>0$ such that

$$
\theta \in[0, \epsilon] \Longrightarrow f^{\prime}(\theta) \geq \eta>0, \quad \eta(1-D) \geq 1,
$$

then the CML (3.3) is persistent.

Proof. (i) For all $\theta_{j}^{t} \in(0, \epsilon]$, using the mean value theorem, there exists $\zeta_{j}^{t} \in\left[0, \theta_{j}^{t}\right]$ such that

$$
\begin{aligned}
\theta_{j}^{t+1} & =(1-D) \theta_{j}^{t}+\left(\frac{D}{2}\right)\left[\theta_{j+1}^{t}+\theta_{j-1}^{t}\right]+f^{\prime}\left(\zeta_{j}^{t}\right) \theta_{j}^{t} \\
& \geq(1-D) \theta_{j}^{t}+\left(\frac{D}{2}\right)\left[\theta_{j+1}^{t}+\theta_{j-1}^{t}\right] .
\end{aligned}
$$

Applying the procedure of Proposition 3.3, then

$$
\theta_{j}^{t+1} \geq \phi_{j}^{t+1} \equiv(1-D) \theta_{j}^{t}+\left(\frac{D}{2}\right)\left[\theta_{j+1}^{t}+\theta_{j-1}^{t}\right] .
$$


The right-hand side is a circulant matrix whose eigenvalues are known and its maximum eigenvalue is a unity, thus $\phi_{j}^{t+1}=\phi_{j}^{0} \equiv \theta_{j}^{0} \Rightarrow \theta_{j}^{t+1} \geq \theta_{j}^{0}>0$.

(ii) For the CML (3.3), the conditions of Proposition 3.3 imply that $\theta_{j}^{t+1} \geq$ $(1-D) f\left(\theta_{j}^{t}\right)$. If $\theta_{j}^{t} \in(0, \epsilon]$, then, using the mean value theorem, we get (so long as $\theta_{j}^{s} \in(0, \epsilon]$ for all $s \leq t$, otherwise $\theta_{j}^{s}>\epsilon$ which implies persistence)

$$
\theta_{j}^{t+1} \geq(1-D) \eta \theta_{j}^{t} \geq[(1-D) \eta]^{t} \theta_{j}^{0}>\theta_{j}^{0}>0
$$

The proof of CML (2.9) is identical.

Applying the above results for the circle map $(\Omega=0)$

$$
f=a \theta-\frac{k}{2 \pi \sin (2 \pi \theta)},
$$

we get that the circle map CML is persistent if

$$
a-k>1 \text {. }
$$

4. Conclusion. The circle maps have many applications in cardiac arrhythmias, ecology, number theory, and so forth. Here, spatial effects are included, so a CML is formed where the local interactions are given by circle maps. Both stability and synchronization, using bounded control and persistence, were studied.

\section{REFERENCES}

[1] V. Afraimovich and B. Fernandez, Topological properties of linearly coupled expanding map lattices, Nonlinearity 13 (2000), no. 4, 973-993.

[2] F. Bagnoli, L. Baroni, and P. Palmerini, Synchronization and directed percolation in coupled map lattices, Phys. Rev. E 59 (1999), 409-416.

[3] S. Barnett, Matrices, Oxford Applied Mathematics and Computing Science Series, The Clarendon Press, Oxford University Press, New York, 1990.

[4] D. Bernard, Turbulence for (and by) amateurs, preprint, 2000, http://fr.arxiv. org/abs/cond-mat/0007106.

[5] N. Chatterjee and N. Gupte, Synchronisation in coupled sine circle maps, preprint, 1995, http://arxiv.org/abs/patt-sol/9510001.

[6] S.-N. Chow and W. Shen, Dynamics in a discrete Nagumo equation: spatial topological chaos, SIAM J. Appl. Math. 55 (1995), no. 6, 1764-1781.

[7] L. Glass, Cardiac arrhythmias and circle maps - a classical problem, Chaos 1 (1991), no. 1, 13-19.

[8] R. O. Grigoriev and H. G. Schuster, A Solvable model for spatiotemporal chaos, preprint, 1997, http://arxiv.org/abs/chao-dyn/9706008.

[9] K. Kaneko (ed.), Theory and Applications of Coupled Map Lattices, Nonlinear Science: Theory and Applications, John Wiley \& Sons, Chichester, 1993.

[10] T. Kwok-keung Au and X.-S. Lin, Off-center reflections: caustics and chaos, Experiment. Math. 10 (2001), no. 2, 287-302.

[11] M. Leo, R. A. Leo, and G. Soliani, A merry-go-round with the circle map, primes and pseudoprimes, preprint, 1999, http://arxiv.org/abs/math.HO/9912079. 
[12] J. Vandermeer, L. Stone, and B. Blasius, Categories of chaos and fractal basin boundaries in forced predator-prey models, Chaos Solitons Fractals 12 (2001), no. 2, 265-276.

[13] D. H. Zanette, Structures and propagation in globally coupled systems with time delays, preprint, 2000, http://arxiv.org/abs/cond-mat/0003174.

E. Ahmed: Mathematics Department, Faculty of Science, Mansoura University, Mansoura 35516, Egypt

Current address: Department of Mathematics and Computer Since, Faculty of Science, United Arab Emirates University, Al-Ain P.O. Box 17551, United Arab Emirates

A. S. Hegazi: Mathematics Department, Faculty of Science, Mansoura University, Mansoura 35516, Egypt 


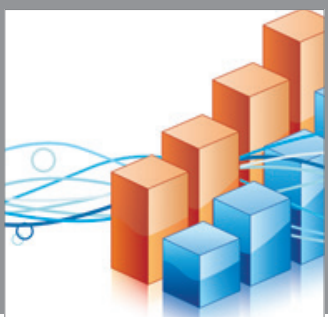

Advances in

Operations Research

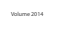



\section{The Scientific} World Journal
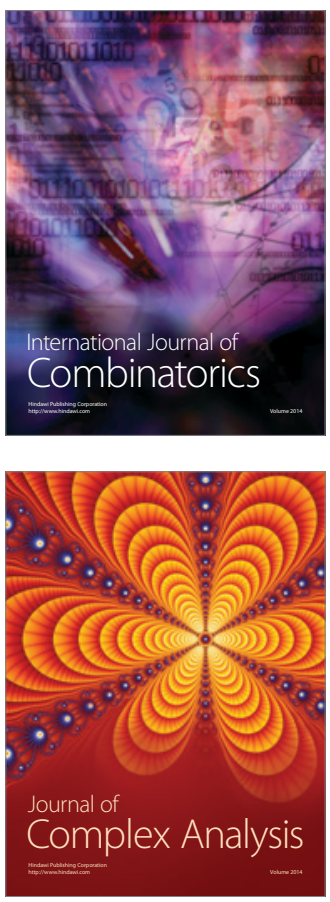

International Journal of

Mathematics and

Mathematical

Sciences


Journal of

Applied Mathematics
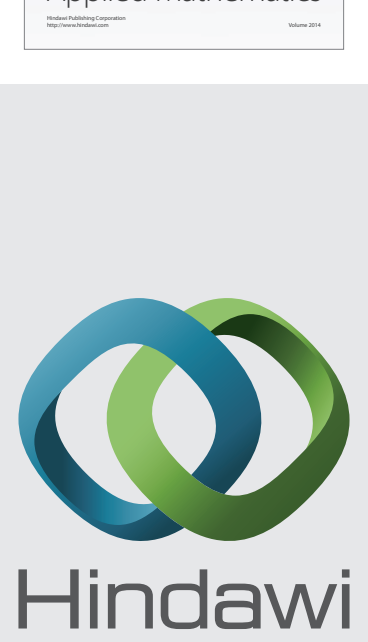

Submit your manuscripts at http://www.hindawi.com


Mathematical Problems in Engineering


Journal of

Function Spaces
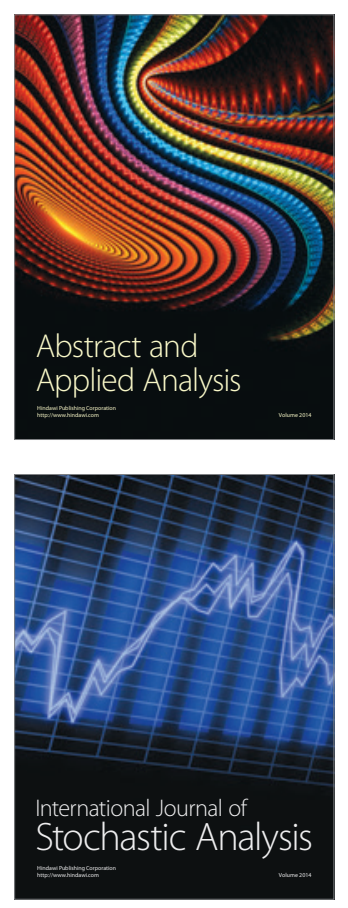

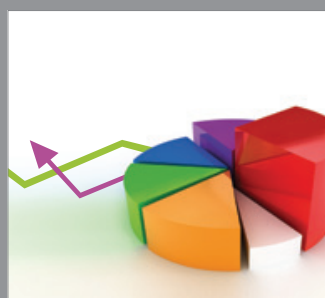

ournal of

Probability and Statistics

Promensencen
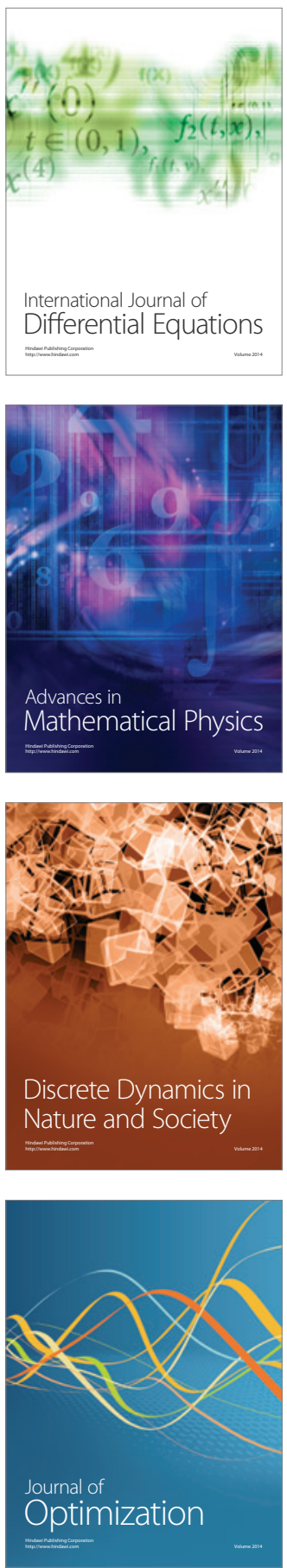